\title{
Nanoscale imaging magnetometry with diamond spins under ambient conditions
}

\author{
Gopalakrishnan Balasubramanian ${ }^{1}$, I. Y. Chan ${ }^{2} \dagger$, Roman Kolesov $^{1}$, Mohannad Al-Hmoud ${ }^{1}, J_{u l i a}$ Tisler $^{1}$, Chang Shin $^{3}$, \\ Changdong Kim ${ }^{3}$, Aleksander Wojcik ${ }^{3}$, Philip R. Hemmer ${ }^{3}$, Anke Krueger ${ }^{4}$, Tobias Hanke ${ }^{5}$, Alfred Leitenstorfer ${ }^{5}$, \\ Rudolf Bratschitsch ${ }^{5}$, Fedor Jelezko ${ }^{1} \&$ Jörg Wrachtrup ${ }^{1}$
}

\begin{abstract}
Magnetic resonance imaging and optical microscopy are key technologies in the life sciences. For microbiological studies, especially of the inner workings of single cells, optical microscopy is normally used because it easily achieves resolution close to the optical wavelength. But in conventional microscopy, diffraction limits the resolution to about half the wavelength. Recently, it was shown that this limit can be partly overcome by nonlinear imaging techniques $^{1,2}$, but there is still a barrier to reaching the molecular scale. In contrast, in magnetic resonance imaging the spatial resolution is not determined by diffraction; rather, it is limited by magnetic field sensitivity, and so can in principle go well below the optical wavelength. The sensitivity of magnetic resonance imaging has recently been improved enough to image single cells ${ }^{3,4}$, and magnetic resonance force microscopy ${ }^{5}$ has succeeded in detecting single electrons ${ }^{6}$ and small nuclear spin ensembles ${ }^{7}$. However, this technique currently requires cryogenic temperatures, which limit most potential biological applications ${ }^{8}$. Alternatively, single-electron spin states can be detected optically ${ }^{9,10}$, even at room temperature in some systems ${ }^{11-14}$. Here we show how magneto-optical spin detection can be used to determine the location of a spin associated with a single nitrogen-vacancy centre in diamond with nanometre resolution under ambient conditions. By placing these nitrogen-vacancy spins in functionalized diamond nanocrystals, biologically specific magnetofluorescent spin markers can be produced. Significantly, we show that this nanometre-scale resolution can be achieved without any probes located closer than typical cell dimensions. Furthermore, we demonstrate the use of a single diamond spin as a scanning probe magnetometer to map nanoscale magnetic field variations. The potential impact of single-spin imaging at room temperature is far-reaching. It could lead to the capability to probe biologically relevant spins in living cells.
\end{abstract}

The nitrogen-vacancy centre in diamond is a unique solid state system that allows ultrasensitive and rapid detection of single electronic spin states under ambient conditions ${ }^{12}$. The nitrogen-vacancy defect is a naturally occurring impurity that is responsible for the pink colouration of diamond crystals when present in high concentration. It was demonstrated that this colour centre can be produced in diamond nanocrystals by electron irradiation. Fluorescing nitrogen-vacancy diamond nanocrystals can be used as markers for bioimaging applications ${ }^{15}$. Such markers have attracted widespread interest because of their unprecedented photostability and non-toxicity $^{16,17}$. It was recognized recently that the magnetic properties of such fluorescent labels can in principle be used for novel microscopy ${ }^{18,19}$. Here we demonstrate the realization of a magneto-optic microscope using nitrogen-vacancy diamond as the magnetic fluorescent label that moreover does not bleach or blink.

Figure $1 \mathrm{c}$ and $\mathrm{d}$ show the fluorescence and atomic force microscope image of nanocrystals containing nitrogen-vacancy defects. By careful choice of irradiation doses, we were able to control the number of nitrogen-vacancy centres per nanocrystal. The particular sample presented in Fig. 1 has on average a single nitrogen-vacancy defect per $40 \mathrm{~nm}$ nanocrystal (confirmed by fluorescence correlation measurements, Fig. 1e).

The energy level scheme and structure of the nitrogen-vacancy defect is shown in Fig. 1a and b. Two out of six electrons of the centre are unpaired, forming an electron spin triplet in the electronic ground and first excited state. Broadband optical excitation of the centre polarizes it by optical pumping into the $m_{s}=0$ spin sublevel. Laser-assisted detection of the spin state of a single nitrogen-vacancy centre makes use of differences in the absorption and emission properties of the spin sublevels. Specifically, the spin sublevel with magnetic quantum number $m_{s}=0$ (bright state) scatters $\sim 30 \%$ more photons than $m_{s}= \pm 1$ states. Hence, when a resonant microwave field induces magnetic dipole transitions between these electronic spin sublevels, it destroys the optically pumped spin polarization, resulting in a significant decrease of the nitrogen-vacancy centre fluorescence. An example of such an optically detected electron spin resonance (ESR) spectrum of a single nitrogen-vacancy electronic spin is shown in Fig. 1f.

The spin Hamiltonian of the nitrogen-vacancy defect (neglecting electron-nuclear spin coupling) can be written as the sum of zero-field and Zeeman terms, $H=D\left(S_{z}^{2}-(1 / 3)[S(S+1)]\right)+E\left(S_{x}^{2}-S_{y}^{2}\right)+$ $g \mu_{\mathrm{B}} \mathbf{B} \cdot \mathbf{S}$, where $D$ and $E$ are zero-field splitting parameters, $S=1, \mu_{\mathrm{B}}$ is the Bohr magneton and $g$ is the electron $g$-factor $(g=2.0)$. Owing to the magnetic dipole interaction between the two unpaired electrons even at zero external magnetic field, the sublevels $m_{s}=0$ and $m_{s}= \pm 1$ are separated $(D=2,870 \mathrm{MHz})$. Owing to symmetry, the $m_{s}= \pm 1$ sublevels of the nitrogen-vacancy defect are degenerate at zero magnetic field $(E=0)$, resulting in a single resonance line appearing in the ESR spectrum (Fig. 1f). An external magnetic field lifts the degeneracy of $m_{s}= \pm 1$, leading to the appearance of two lines. By measuring the positions of the ESR resonances $\omega_{1}$ and $\omega_{2}$, it is possible to calculate the magnitude of the external field $B$ according to $(g \mu B)^{2}=$ $(1 / 3)\left(\omega_{1}^{2}+\omega_{2}^{2}-\omega_{1} \omega_{2}-D^{2}\right)-E^{2}$ (see Methods for details).

From the above-mentioned relations, it can be seen that, when combined with nano-positioning instrumentation, the single spin associated with a nitrogen-vacancy defect can be used as an atomsized scanning probe vector magnetometer. Similarly, when placed in

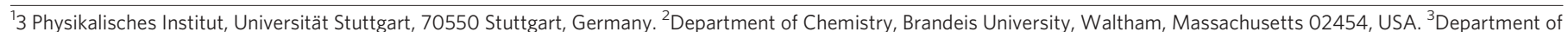

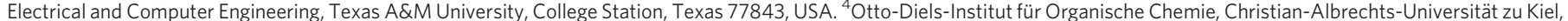

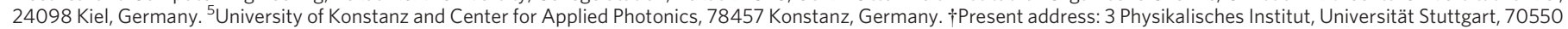
Stuttgart, Germany. 
an inhomogeneous magnetic field with a known field gradient, the defect can be used as a magneto-optical spin marker for subopticalwavelength tagged imaging. As a demonstration, two-dimensional spin imaging experiments were performed using a single nitrogenvacancy centre and the highly inhomogeneous magnetic field produced by the magnetic tip of an atomic force microscope (AFM). The experimental set-up is shown in Fig. 2a. A commercial AFM was combined with a confocal microscope. The magnetic probe, commonly used in magnetic force microscopy, consists of a sharp silicon tip coated with $30 \mathrm{~nm}$ of magnetic material: the exact magnetic field profile of the cantilever is not known a priori, and must be determined. For this, we have used our single-spin nitrogen-vacancy magnetometer. The magnetic cantilever was first placed at a known distance from the diamond nanocrystal, and the magnetic field experienced by the single nitrogen-vacancy centre was recorded in steps (corresponding to several hundred nanometre displacements of the cantilever) by acquiring ESR spectra such as those in Fig. If at each location. The experimentally obtained data points were then fitted using a Lorentzian function, inferred from numerical simulation of the field created by the cantilever (Fig. 2b). This gives the magnetic field profile of the cantilever in one dimension. Similarly, the profile along an orthogonal axis is recorded to give the $\mathbf{a}$

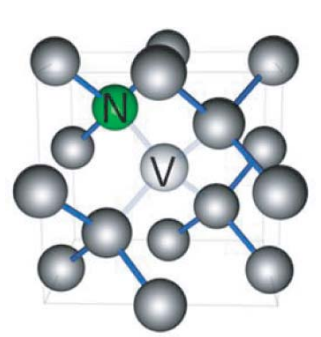

c
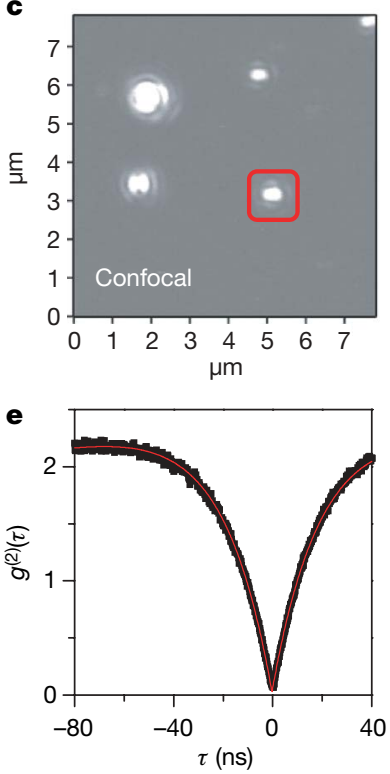

b
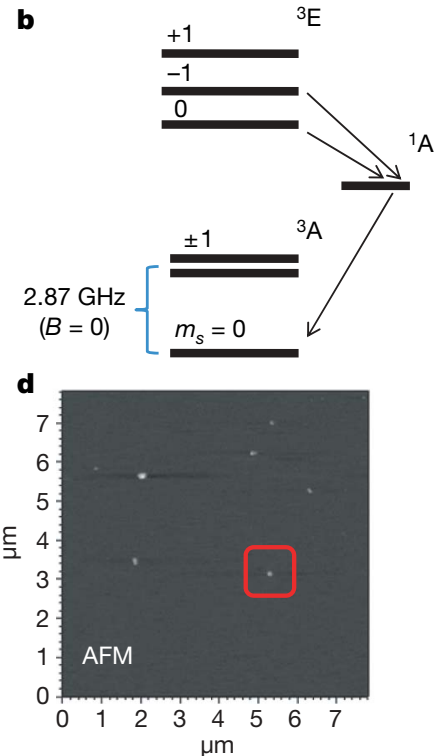

f

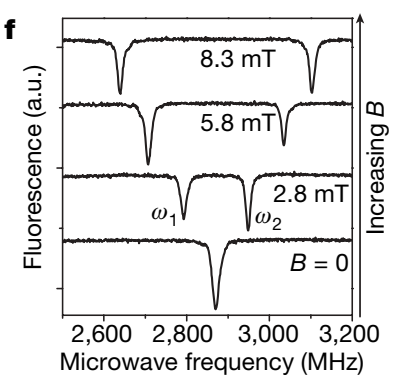

Figure 1 | Nitrogen-vacancy defect in diamond. a, Structure and energy level scheme of the nitrogen-vacancy $(\mathrm{NV})$ defect in diamond. Optical pumping initializes the centre into the $m_{s}=0$ spin state via spin selective shelving into the metastable singlet state, ${ }^{1} \mathrm{~A}$. This state decays preferentially into the $m_{s}=0$ sublevel of the ground state, leading to optically induced spin polarization (more than $>90 \%$ at room temperature). c, d, Simultaneously acquired optical (c) and AFM (d) image of diamond nanocrystals containing single nitrogen-vacancy defects. e, Fluorescence autocorrelation function, confirming that the nanocrystal contains a single nitrogen-vacancy defect. The contrast of $g^{2}(\tau)$ at zero delay time scales as $1 / N$, where $N$ is the number of emitters. f, Optically detected magnetic resonance spectra for a single nitrogen-vacancy defect at increasing magnetic field (from bottom to top). two-dimensional profile as well as the exact position of the nitrogen-vacancy centre.

To visualize the resolving power of our gradient imaging technique, the magnetic cantilever was scanned in the vicinity of a nanocrystal containing a single nitrogen-vacancy defect while simultaneously exciting with a fixed-frequency microwave field. When a confocal image is acquired, each point of the optical image corresponds to a well-defined magnetic field value (as measured in
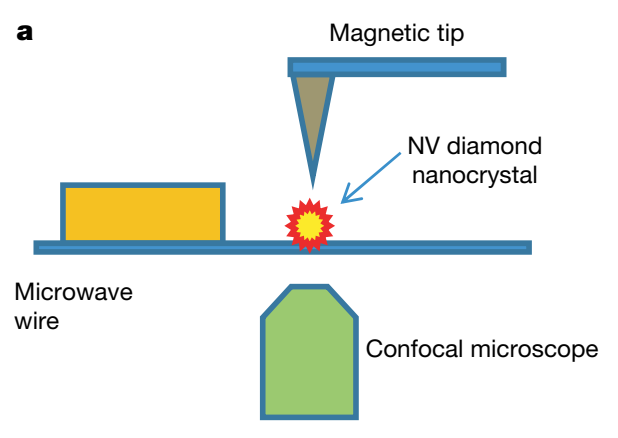

b
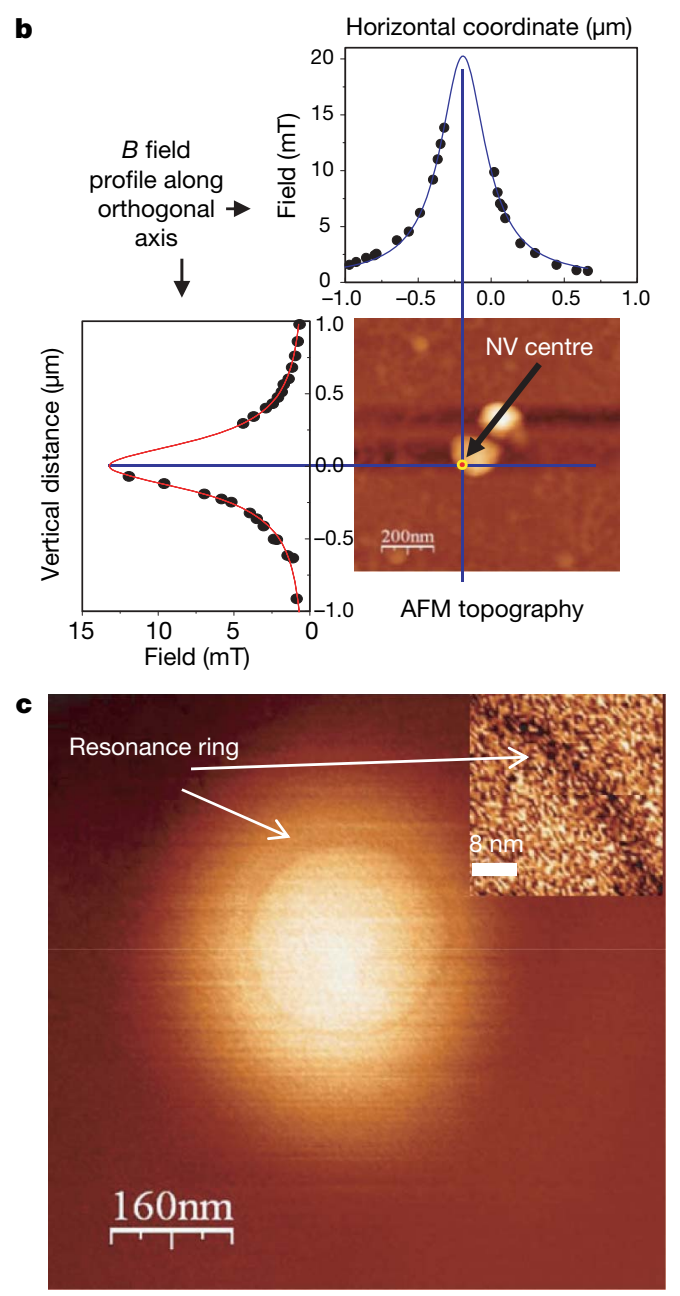

Figure 2 | Gradient imaging with single spins. a, Two-dimensional imaging is achieved using a field gradient created by a magnetic cantilever. $\mathbf{b}$, The experimental profile of the cantilever's magnetic field for two orthogonal axes. The magnetic tip was placed at several points parallel to the blue lines, and the ESR spectra were measured. The calculated (fitted) magnetic field profile allows estimation of the location of the single nitrogen-vacancy centre (shown in the AFM topography). c, Two-dimensional magnetic resonance image of a single nitrogen-vacancy centre, showing resonance rings corresponding to a magnetic field of $3 \mathrm{mT}$ (resonance frequency of $2,780 \mathrm{MHz}$ ). Inset, an enlarged section of a ring with a width of approximately $5 \mathrm{~nm}$. 
the previous experiment). At particular positions (pixels) when the microwave frequency is resonant with the corresponding spin sublevel splitting, the fluorescence intensity is reduced. This results in a dark ring (Fig. 2), which marks a two-dimensional cut through the $B$ field corresponding to a constant magnetic field projection along the nitrogen-vacancy quantization axis. The width of the rings is given by the magnetic resonance linewidth divided by the field gradient $\left(80 \mu \mathrm{T} \mathrm{nm}^{-1}\right)$. This ring width also defines the ultimate resolution limit of this technique for spin imaging. Rings with $5 \mathrm{~nm}$ width are observed, and shown in Fig. $2 \mathrm{c}$ inset. Note that this width is smaller than the sizes of both the magnetic tip and the diamond nanocrystal, and is only possible because a single nitrogen-vacancy centre is localized to a fraction of a nanometre in the diamond lattice. The dark ring shown in Fig. 2c is only seen if simultaneously the nitrogenvacancy axis is oriented vertically, and the magnetic field is radially symmetric. This special case was selected to simplify understanding of the technique. It is interesting to note that a vibrating cantilever (a.c. mode AFM was used in all the experiments) induces significant line broadening when the magnetic cantilever comes very close to the spin (see Supplementary Information).

Being an atomic-sized non-perturbing magnetic field sensor, the single nitrogen-vacancy centre can be incorporated into the cantilever instead of a magnetic coating, and used as a scanning probe magnetometer to achieve subwavelength imaging resolution. To demonstrate the feasibility of this approach, we attached a nanocrystal containing a single nitrogen-vacancy centre to the tip of a cantilever, and used it to profile the magnetic field produced by a nanometre-sized magnetic structure. Details of the set-up are shown in Fig. 3a. Microwaves are tuned into resonance with the nitrogen-vacancy spin at the tip of the cantilever (see Fig. 3b) for a particular magnetic field projection. Hence the resonance conditions in the vicinity of the magnetic nanostructures are satisfied along well-defined lines of constant $B_{z}$, where $z$ is along the nitrogen-vacancy quantization axis. Figure $3 c$ shows a magneto-optical image of a triangular magnetic structure obtained with a single nitrogen-vacancy defect as light source (as expected, the structure appears as a shadow in our detection geometry). The narrow dark line close to the corner represents spatial regions where the conditions for magnetic resonance of the nitrogen-vacancy centre on the tip are fulfilled $\left(B_{z}=5 \mathrm{mT}\right)$. Note that the image represents raw data acquired in just 4 minutes. The magnetic field resolution is given by the width of the dark lines, which are about $20 \mathrm{~nm}$, multiplied by the magnetic field gradient of $25 \mu \mathrm{T} \mathrm{nm}^{-1}$ (measured by recording several resonance lines at different microwave frequencies, data not shown). It corresponds to a measurement resolution of $0.5 \mathrm{mT}$. The limiting factor here is oscillatory motion of the nanodiamond attached to the AFM tip (see Methods for details).

The resolution could be significantly improved by phase locking of the detection system to the oscillatory motion of the cantilever, and using echo-based techniques with an echo period matched to a single oscillation period of the cantilever. This essentially corresponds to measuring a.c. instead of d.c. magnetic fields. The advantage of using echoes is that the effective ESR linewidth is narrower than the inhomogeneous linewidth ${ }^{20}$, and for a long spin phase memory time $T_{2}$ is effectively given by the AFM vibration frequency, which is $100 \mathrm{kHz}$ for standard AFM cantilevers. Hence we expect an improvement of field measurement accuracy by a factor of $150(3 \mu \mathrm{T})$ using this technique. For the magnetic field gradient caused by the structure imaged in Fig. 3c, this would correspond to subnanometre spatial resolution.

Ultrasensitive magnetometry with single spins in diamond not only allows high spatial resolution imaging, but also might be applied to image single external spins under ambient conditions. Here the magnetic sublevels of the nitrogen-vacancy centre are shifted by the magnetic fields produced by (for example) other single electron or nuclear magnetic dipoles in nearby molecules. To show the feasibility of single electron and nuclear spin detection, we estimate the ultimate sensitivity limit of a scanning spin microscope based on nitrogen-vacancy centres in diamond. The magnetic field created by a single electron spin located at distance $r$ from the nitrogen-vacancy spin is $B^{\text {dip }}=\left(\mu_{0} \mu / 4 \pi\right) \sqrt{3 \cos ^{2} \theta+1} / r^{3}$, where is $\mu$ the single spin magnetic moment, and $\theta$ is the angle between the vector connecting the two spins and the vector of the external magnetic field. When we substitute $\mu=-(1 / 2) g_{\mathrm{e}} \mu_{\mathrm{B}} \approx 10^{-23} \mathrm{JT}^{-1}$, and $\mu_{0} / 4 \pi \approx 10^{-7} \mathrm{NA}^{-2}$, a field of $10^{-5} \mathrm{~T}$ can be obtained for a distance between the electron and nitrogen-vacancy spins of $5 \mathrm{~nm}$. For the nitrogen-vacancy centre this gives up to $0.3 \mathrm{MHz}$ of ESR frequency shift, which is within the projected detection limit for the single nitrogen-vacancy nanocrystals used in this demonstration. Imaging single nuclear spins is more challenging, as the lower nuclear magnetic moment results in fields three orders of magnitude lower $\left(10^{-8} \mathrm{~T}\right)$. This value corresponds to a kilohertz shift of the nitrogen-vacancy resonance.

In general, the sensitivity of our nitrogen-vacancy magnetometer is determined by the linewidth of the ESR transition. Experiments presented here were carried out using continuous wave (c.w.) ESR
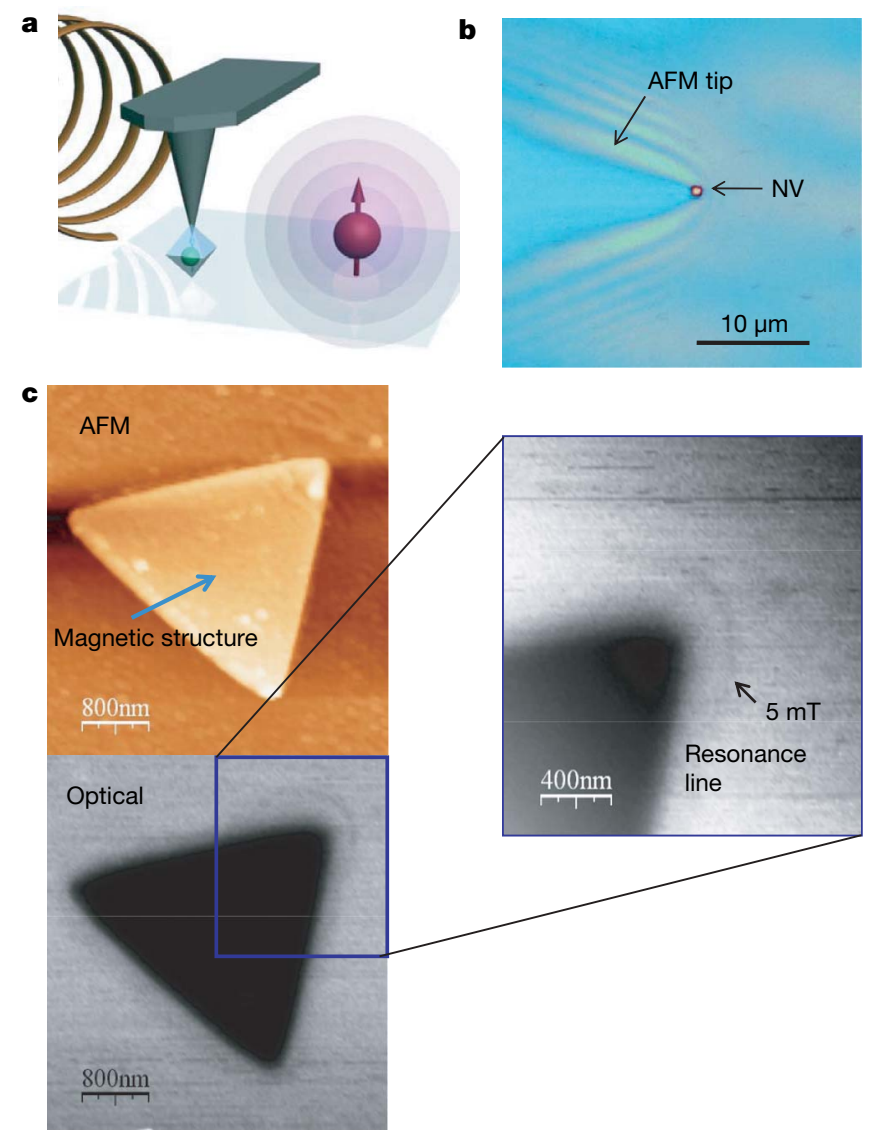

Figure 3 | Scanning probe magnetometry. a, Diagram of the magnetic field imaging experiment. A nanoscale magnetic particle (red) is imaged with a single nitrogen-vacancy defect (green, within the blue nanocrystal) fixed at the scanning probe tip (black). b, Optical image of a diamond nanocrystal attached to an AFM tip (view from the bottom). The scattered light image of the tip is overlapped with the fluorescence image of the nanocrystal. The bright spot (arrowed) represents fluorescence of a single nitrogen-vacancy defect. Fluorescence autocorrelation function (data not shown) shows a pronounced antibunching dip, indicating a single nitrogen-vacancy defect in the nanocrystal on the AFM tip. c, Field reconstruction using the scanning probe single spin magnetometer. Top left, an AFM image of a nickel magnetic nanostructure prepared by electron beam lithography; bottom left, a magneto-optical image of the same structure, recorded using a single nitrogen-vacancy centre on the AFM tip as light source and magnetometer. Inset (right), the fluorescence signal from the scanned nitrogen-vacancy centre attached to the apex of the AFM tip when resonant microwaves at $2,750 \mathrm{MHz}$ are applied (the arrowed point corresponds to $5 \mathrm{mT}$ resonance line with the magnetic field tilted by $45^{\circ}$ relative to the nitrogen-vacancy axis). 
and technical grade diamonds. Hence the linewidth of the spin resonance line (a few $\mathrm{MHz}$ ) was limited by fast $(\mu \mathrm{s})$ decoherence and microwave-field-induced broadening. However, it was recently shown that the phase memory time for ultrapure diamond reaches one millisecond when echo-based techniques are used for detection $^{21}$. This corresponds to a linewidth of the order of $0.3 \mathrm{kHz}$. Taking this value, single nuclear spins can be detected at $5 \mathrm{~nm}$ distance under ambient conditions ${ }^{22}$. As these spin linewidths were obtained under ambient conditions, this approach will potentially enable the use of nitrogen-vacancy defects in diamond nanocrystals as a probe for intracellular electron (and possibly nuclear) spin imaging.

\section{METHODS SUMMARY}

Magnetic imaging and magnetometry experiments were performed using a home-built scanning confocal microscope combined with an AFM (MFP-3D Asylum Research). Nitrogen-vacancy defects were excited with a frequency doubled c.w. Nd:YAG laser (Coherent Compass) focused on to the sample with a high NA objective (Olympus PlanAPO, NA = 1.35). Luminescence light was collected by the same objective and filtered from the excitation light using a dichroic beamsplitter (640 DCXR, Chroma) and long-pass filter (647 LP, Chroma). Photon counting of the filtered light was performed using two avalanche photodiodes (SPQR-14, Perkin-Elmer). Fluorescence autocorrelation histograms were recorded using a fast multichannel analyser (Fastcomtec, P7889). Optically detected magnetic resonance measurements were performed using a commercial microwave source (Rhode \& Schwarz GmbH, SMIQ 03) amplified by a travelling wave tube amplifier (Hughes $8020 \mathrm{H}$ ). Commercially available magnetic cantilevers (Team Nanotec) were used for generation of high magnetic field gradients. UV curing glue (Thorlabs) was used to attach diamond nanocrystals to the AFM tip for the scanning probe magnetometry.

Full Methods and any associated references are available in the online version of the paper at www.nature.com/nature.

\section{Received 23 April; accepted 18 July 2008.}

1. Willig, K. I., Rizzoli, S. O., Westphal, V., Jahn, R. \& Hell, S. W. STED microscopy reveals that synaptotagmin remains clustered after synaptic vesicle exocytosis. Nature 440, 935-939 (2006).

2. Betzig, E. et al. Imaging intracellular fluorescent proteins at nanometer resolution. Science 313, 1642-1645 (2006).

3. Aguayo, J. B., Blackband, S. J., Schoeniger, J., Mattingly, M. A. \& Hintermann, M. Nuclear-magnetic-resonance imaging of a single cell. Nature 322, 190-191 (1986).

4. Ciobanu, L., Seeber, D. A. \& Pennington, C. H. 3D MR microscopy with resolution $3.7 \mu \mathrm{m}$ by $3.3 \mu \mathrm{m}$ by $3.3 \mu \mathrm{m}$. J. Magn. Reson. 158, 178-182 (2002).

5. Rugar, D., Yannoni, C. S. \& Sidles, J. A. Mechanical detection of magnetic resonance. Nature 360, 563-566 (1992).

6. Rugar, D., Budakian, R., Mamin, H. J. \& Chui, B. W. Single spin detection by magnetic resonance force microscopy. Nature 430, 329-332 (2004).
7. Mamin, H. J., Poggio, M., Degen, C. L. \& Rugar, D. Nuclear magnetic resonance imaging with 90-nm resolution. Nature Nanotechnol. 2, 301-306 (2007).

8. Glover, P. \& Mansfield, P. Limits to magnetic resonance microscopy. Rep. Prog. Phys. 65, 1489-1511 (2002).

9. Kohler, J. et al. Magnetic resonance of a single molecular spin. Nature 363, 242-244 (1993)

10. Wrachtrup, J., von Borczyskowski, C., Bernard, J., Orrit, M. \& Brown, R. Optical detection of magnetic resonance in a single molecule. Nature 363, 244-245 (1993).

11. Hanson, R., Dobrovitski, V. V., Feiguin, A. E., Gywat, O. \& Awschalom, D. D. Coherent dynamics of a single spin interacting with an adjustable spin bath. Science 320, 352-355 (2008).

12. Gruber, A. et al. Scanning confocal optical microscopy and magnetic resonance on single defect centers. Science 276, 2012-2014 (1997).

13. Epstein, R. J., Mendoza, F. M., Kato, Y. K. \& Awschalom, D. D. Anisotropic interactions of a single spin and dark-spin spectroscopy in diamond. Nature Phys. 1, 94-98 (2005)

14. Childress, L. et al. Coherent dynamics of coupled electron and nuclear spin qubits in diamond. Science 314, 281-285 (2006).

15. Fu, C. C. et al. Characterization and application of single fluorescent nanodiamonds as cellular biomarkers. Proc. Natl Acad. Sci. USA 104, 727-732 (2007).

16. Liu, K. K., Cheng, C. L., Chang, C. C. \& Chao, J. I. Biocompatible and detectable carboxylated nanodiamond on human cell. Nanotechnology 18, 325102 (2007).

17. Neugart, F. et al. Dynamics of diamond nanoparticles in solution and cells. Nano Lett. 7, 3588-3591 (2007).

18. Chernobrod, B. M. \& Berman, G. P. Spin microscope based on optically detected magnetic resonance. J. Appl. Phys. 97, 014903 (2005).

19. Kuhn, S., Hettich, C., Schmitt, C., Poizat, J. P. H. \& Sandoghdar, V. Diamond colour centres as a nanoscopic light source for scanning near-field optical microscopy. J. Microsc. 202, 2-6 (2001).

20. Taylor, J. M. et al. High-sensitivity diamond magnetometer with nanoscale resolution. Nature Phys. (in the press); preprint at 〈http://arXiv.org/abs/ 0805.1367v1) (2008).

21. Gaebel, T. et al. Room-temperature coherent coupling of single spins in diamond. Nature Phys. 2, 408-413 (2006).

22. Maze, J. R. et al. Nanoscale magnetic sensing with an individual electronic spin in diamond. Nature doi:10.1038/nature07279 (this issue).

Supplementary Information is linked to the online version of the paper at www.nature.com/nature.

Acknowledgements We thank M. D. Lukin for drawing our attention to advanced echo-based techniques, and R. Kamella for technical assistance. This work was supported by the EU (QAP, EQUIND, NANO4DRUGS, NEDQIT), DFG (SFB/TR21 and FOR730) and Landesstiftung BW.

Author Contributions G.B., I.Y.C, R.K., M.A.-H., J.T., C.S., C.K., A.W., J.W. and F.J. performed the experiments; A.K. prepared diamond nanocrystals; T.H., A.L. and R.B. prepared magnetic nanostructures; P.R.H., J.W. and F.J. designed and coordinated the experiments; and F.J. wrote the paper. All authors discussed the results, analysed the data and commented on the manuscript.

Author Information Correspondence and requests for materials should be addressed to F.J. (f.jelezko@physik.uni-stuttgart.de). 


\section{METHODS}

Vector magnetometry using a single nitrogen-vacancy defect electron spin. The resonance frequencies of the $m_{s}=0 \leftrightarrow \pm 1$ spin transitions of a spin- 1 system allow one to extract the magnitude of the local magnetic field and, under some approximations, the angle between the magnetic field and the symmetry axis of the system. The spin Hamiltonian of an $S=1$ system having a distorted $C_{3 v}$ symmetry is given by the following expression:

$$
H=\mu_{\mathrm{B}} g \mathbf{B} \cdot \mathbf{S}+D\left(S_{z}^{2}-S(S+1) / 3\right)+E\left(S_{x}^{2}-S_{y}^{2}\right)
$$

where $D$ and $E$ are the zero-field splitting parameters, $S=1, \mu_{\mathrm{B}}$ is the Bohr magneton, $g=2$ is the $g$-factor, and $\mathbf{B}$ is the external magnetic field. Imperfect axial symmetry is reflected by the asymmetry parameter $E$. These parameters unambiguously determine the natural local coordinate system, with the $z$ axis being along the axis of the nitrogen-vacancy centre and $x$ and $y$ axes being along the principal axes of the distortion ellipsoid. In such a coordinate system, it is convenient to describe the magnetic field by its magnitude $B$ and the two angles, $\theta$ (polar) and $\varphi$ (azimuthal). All parameters $B, \theta$ and $\varphi$ can be obtained from analysis of the ESR spectrum. The positions of spin levels are given by the solutions of the characteristic equation:

$x^{3}-\left(\frac{D^{2}}{3}+E^{2}+\beta^{2}\right) x-\frac{\beta^{2}}{2}\left(D \cos 2 \theta+2 E \cos 2 \varphi \sin ^{2} \theta\right)-\frac{D}{6}\left(4 E^{2}+\beta^{2}\right)+\frac{2 D^{3}}{27}=0$

where $\beta=\mu_{\mathrm{B}} g B$. Denoting the frequency of the $S_{z}=0$ state as $x_{0}$, one finds that the positions of the $S_{z}= \pm 1$ states are given by $x_{ \pm}=x_{0}+v_{0 \pm}$, where $v_{0 \pm}$ are the experimentally measured frequencies of $0 \leftrightarrow \pm 1$ spin transitions. Since $x_{ \pm}$must satisfy the above-mentioned equation, it is possible to obtain three equations for the four unknowns $\left(x_{0}, B, \theta\right.$ and $\left.\varphi\right)$, two of which, $\theta$ and $\varphi$, form a unique combination $\Delta=D \cos 2 \theta+2 E \cos 2 \varphi \sin ^{2} \theta$. This set of equations gives the following solutions for $\beta$ and $\Delta$ :

$$
\begin{aligned}
& \beta^{2}=\frac{1}{3}\left(v_{1}^{2}+v_{2}^{2}-v_{1} v_{2}-D^{2}\right)-E^{2}, \\
& \Delta=\frac{7 D^{3}+2\left(v_{1}+v_{2}\right)\left(2\left(v_{1}^{2}+v_{2}^{2}\right)-5 v_{1} v_{2}-9 E^{2}\right)-3 D\left(v_{1}^{2}+v_{2}^{2}-v_{1} v_{2}+9 E^{2}\right)}{9\left(v_{1}^{2}+v_{2}^{2}-v_{1} v_{2}-D^{2}-3 E^{2}\right)}
\end{aligned}
$$

Since for nitrogen-vacancy centres $D \gg E, \Delta \approx D \cos 2 \theta$. Thus, knowing the zerofield splitting parameters and the frequencies of the $0 \leftrightarrow \pm 1$ ESR resonances, one can find $B$ and $\theta$. The solution of the inverse problem of finding the two ESR frequencies given the known $B$ and $\theta$ is presented in Supplementary Information. Modelling the magnetic field of the cantilever. The magnetic field of a tip having a ferromagnetic coating was simulated in the following manner. The surface of the tip is assumed to be an axially symmetric cone with a round apex. It can be simulated by the following simple analytical formula:

$$
h=r \tanh \frac{r}{2 r_{0}}
$$

where $h$ is the height of the surface point above the apex, $r$ is the radial coordinate, and $r_{0}$ is the curvature radius of the rounded apex of the tip. It is assumed that the tip surface is covered with a thin layer of ferromagnetic material. The magnetic field produced by an infinitely small element of the surface was approximated as that of a magnetic dipole. The magnetization of the surface is assumed to be uniform and the direction of the magnetization of each surface element assumed the same. The contributions of all surface elements were then integrated over the surface of the tip. We are interested in the magnetic field in a plane somewhat below the tip apex and perpendicular to the tip axis. In the simplest case of the magnetization pointing along the tip axis, the magnetic field has only radial and axial components. The typical result of a simulation is shown in Supplementary Information. It justifies the Lorentzian field distribution model used to find the two-dimensional position of the nitrogen-vacancy centre. 